\title{
ON TIME-SPLITTING METHODS FOR NONLINEAR SCHRÖDINGER EQUATION WITH HIGHLY OSCILLATORY POTENTIAL
}

\author{
CHUNMEI SU AND XIAOFEI ZHAO
}

\begin{abstract}
In this work, we consider the numerical solution of the nonlinear Schrödinger equation with a highly oscillatory potential (NLSE-OP). The NLSE-OP is a model problem which frequently occurs in recent studies of some multiscale dynamical systems, where the potential introduces wide temporal oscillations to the solution and causes numerical difficulties. We aim to analyze rigorously the error bounds of the splitting schemes for solving the NLSE-OP to a fixed time. Our theoretical results show that the Lie-Trotter splitting scheme is uniformly and optimally accurate, while the Strang splitting scheme is not. Our results apply to general dispersive or wave equations with an oscillatory potential. The error estimates are confirmed by numerical results.
\end{abstract}

Keywords: nonlinear Schrödinger equation, highly oscillatory potential, Lie-Trotter splitting, Strang splitting, error estimates, uniformly accurate.

AMS Subject Classification: 65L05, 65L20, 65L70.

\section{INTRODUCTION}

In quantum and plasma physics, to observe and address particular physical phenomenon, parameters of different scales are frequently introduced into the related dispersive or kinetic modelling equations. These parameters (often scaled to be small) cause multiscale behavior in the solution. For example, the non-relativistic limit regime of the Klein-Gordon equation $[2,8]$, the subsonic limit regime of the Zakharov system $[5,6]$ and the strong magnetic field regime of the Vlasov equation $[14,15]$ all induce high temporal oscillations of the corresponding solutions. This kind of problems, usually after some suitable change of variable which filters out the stiffest part in the equation, can be reformulated into a highly oscillatory problem:

$$
\dot{u}(t)=f(t / \varepsilon, u(t)), \quad t>0,
$$

where $\varepsilon \in(0,1]$. The common fact that $\ddot{u}(t)=O(1 / \varepsilon)$ makes standard numerical integration schemes based on finite difference discretization suffering from low efficiency, since the time step has to be restricted by $\varepsilon$. Therefore, efforts have been made in recent research to design uniformly accurate methods aiming to overcome the time step size restriction $[2,6,8,11,14]$. To gain a uniform accuracy which means that the error is independent of $\varepsilon$, the proposed numerical schemes are equipped with multiscale techniques such as asymptotic expansion [2, 6, 12], two-scale formulation [11, 14] or iterative strategy [8]. However, these multiscale techniques would break the intrinsic structure such as symmetry and Hamiltonian in the original model, which lead to numerical schemes missing ideal long-time performance. Moreover, for some problems the multiscale methods may be over sophisticated to some extent.

In this paper, we shall investigate a simple case of (1.1) where the dependence on $t / \varepsilon$ is explicit and linear, by considering the following nonlinear Schrödinger equation with an external highly oscillatory potential (NLSE-OP):

$$
\begin{aligned}
& i \partial_{t} u(\mathbf{x}, t)=\Delta u(\mathbf{x}, t)+\left(V^{\varepsilon}(\mathbf{x}, t)+f\left(|u(\mathbf{x}, t)|^{2}\right)\right) u(\mathbf{x}, t), \quad t>0, \quad \mathbf{x} \in \mathbb{T}^{d}, \\
& u(\mathbf{x}, 0)=u_{0}(\mathbf{x}), \quad \mathbf{x} \in \mathbb{T}^{d} .
\end{aligned}
$$

Here $d=1,2,3, \mathbb{T}=[-\pi, \pi], u:=u(\mathbf{x}, t)$ is the complex-valued unknown, and $u_{0}$ is the given initial value, $V^{\varepsilon}(\mathbf{x}, t)$ is a given real-valued smooth function with dependence of a parameter $0<\varepsilon \leq 1$ 
and $f \in C^{\infty}(\mathbb{R}, \mathbb{R})$ is the given nonlinearity. The potential function $V^{\varepsilon}(\mathbf{x}, t)$ is assumed to oscillate in time with frequency inversely proportional to $\varepsilon$ and

$$
\partial_{t}^{k} V^{\varepsilon}=O\left(\varepsilon^{-k}\right), \quad k \in \mathbb{N}, 0<\varepsilon \ll 1 .
$$

Note that when the highly oscillatory potential $V^{\varepsilon}(\mathrm{x}, t)$ is given for instance as a fast traveling wave:

$$
\varepsilon^{2} \partial_{t t} V^{\varepsilon}-\Delta V^{\varepsilon}=|u|^{2},
$$

the NLSE-OP coupled with the above wave equation form a Hamiltonian system. The model problem (1.2) is essentially motivated from the recent numerical study of the subsonic limit of the Zakharov system [6], where the highly oscillatory potential represents the fast out-going initial layer. Problems with a similar form as (1.2) would also be encountered in situations such as the simultaneously highplasma-frequency and subsonic limit regime of the Klein-Gordon-Zakharov system [7, 21], the NLS formulation of the nonlinear Klein-Gordon equation in the non-relativistic limit regime [8] and also the rapidly rotating regime of the Klein-Gordon equation in Lagrangian coordinates [22]. In case that $V^{\varepsilon}(\mathbf{x}, t)$ could be integrated exactly in time, the operator splitting schemes which are undoubtedly one of the most popular numerical methods for solving Shrödinger type equations, can give promising numerical approximations to (1.2) in terms of accuracy for all $\varepsilon \in(0,1]$. In fact, a multiscale scheme based on a splitting solver for the NLSE-OP displays a uniform accuracy for solving the Zakharov system in the subsonic limit regime [6], where the fast out-going wave type potential $V^{\varepsilon}$ in such case acts only for a very short time.

In this work, we are going to rigorously analyze the error bounds of the classical splitting schemes: the Lie-Trotter splitting and the Strang splitting for solving the NLSE-OP (1.2) with a general highly oscillatory potential (1.3). Note that the torus setup in (1.2) ensures that the potential $V^{\varepsilon}$ never vanishes even if it is a traveling wave. The convergence analysis of splitting methods for Schrödinger type problems has been widely carried out in the literature $[9,13,17,19,23,25]$. By borrowing some of the state-of-the-art techniques, in this work we make special efforts to derive the dependence of the error bound on the wavelength $\varepsilon$ in the potential $V^{\varepsilon}$ up to a fixed time. We shall show through theoretical error estimates and numerical tests that the Lie-Trotter splitting scheme gives uniform first order accuracy in solving (1.2) for all $\varepsilon \in(0,1]$, while the Strang splitting scheme fails to reach its optimal second order accuracy for all $\varepsilon \in(0,1]$. However, thanks to the exact integration of the oscillatory potential, the splitting schemes still give much more accurate approximations than the exponential integrators (or called as trigonometric integrators) [3, 29, 30] in the highly oscillatory regime. Though we focus on the NLSE-OP (1.2), our analysis also applies to general dispersive or wave equations with oscillatory potentials. The extension to the nonlinear Klein-Gordon equation with an oscillatory potential could be potentially used to design uniformly accurate schemes for the Klein-Gordon-Zakharov system in the simultaneously high-plasma-frequency and subsonic limit regime.

The rest of the paper is organized as follows. In Section 2, we present the splitting schemes for solving (1.2) and the corresponding convergence results. Section 3 and Section 4 are devoted to proving the error estimates of the Lie-Trotter splitting scheme and the Strang splitting scheme, respectively. Numerical results are given in Section 5 to illustrate the theoretical results. Conclusions are drawn in Section 6.

\section{SPlitTing SChEmes}

In this section, we shall briefly present the Lie-Trotter splitting scheme and the Strang splitting scheme for solving the NLSE-OP, and then present the main results on the error estimates.

2.1. Schemes and notations. We denote in the following $\tau=\Delta t>0$ and $t_{n}=n \tau$. As is widely used, the time splitting method splits the NLSE-OP (1.2) for some $t=s+t^{\prime}$ into subproblems

$$
\left\{\begin{array}{l}
i \partial_{s} v\left(\mathbf{x}, s+t^{\prime}\right)=\Delta v\left(\mathbf{x}, s+t^{\prime}\right), \quad \mathbf{x} \in \mathbb{T}^{d}, s>0, \\
v\left(\mathbf{x}, t^{\prime}\right)=v_{0}(\mathbf{x}), \quad \mathbf{x} \in \mathbb{T}^{d},
\end{array}\right.
$$


and

$$
\left\{\begin{array}{l}
i \partial_{s} w\left(\mathbf{x}, s+t^{\prime}\right)=\left(V^{\varepsilon}\left(\mathbf{x}, s+t^{\prime}\right)+f\left(\left|w\left(\mathbf{x}, s+t^{\prime}\right)\right|^{2}\right)\right) w\left(\mathbf{x}, s+t^{\prime}\right), \quad \mathbf{x} \in \mathbb{T}^{d}, s>0 \\
w\left(\mathbf{x}, t^{\prime}\right)=w_{0}(\mathbf{x}), \quad \mathbf{x} \in \mathbb{T}^{d}
\end{array}\right.
$$

Note in $(2.2)\left|w\left(\mathbf{x}, s+t^{\prime}\right)\right| \equiv\left|w_{0}(\mathbf{x})\right|$ for all $s \geq 0$, since $V^{\varepsilon}$ and $f$ are real-valued. The exact solutions of the subproblems (2.1) and (2.2) can be written explicitly as

$$
v\left(\mathbf{x}, s+t^{\prime}\right)=\varphi_{T}^{s}\left(v_{0}\right):=\mathrm{e}^{-i s \Delta} v_{0}(\mathbf{x})
$$

and

$$
w\left(\mathbf{x}, s+t^{\prime}\right)=\varphi_{V}^{s, t^{\prime}}\left(w_{0}\right):=\mathrm{e}^{-i \int_{0}^{s} V^{\varepsilon}\left(\mathbf{x}, t^{\prime}+y\right) d y-i s f\left(\left|w_{0}(\mathbf{x})\right|^{2}\right)} w_{0}(\mathbf{x}),
$$

respectively. Setting $u^{0}=u_{0}$. The first order Lie-Trotter splitting scheme reads as

$$
u^{n}=\Phi_{L}^{\tau, t_{n-1}}\left(u^{n-1}\right), \quad n \geq 1, \quad \Phi_{L}^{\tau, t^{\prime}}(\eta):=\varphi_{T}^{\tau} \circ \varphi_{V}^{\tau, t^{\prime}}(\eta)
$$

and the second order Strang splitting scheme reads as

$$
u^{n}=\Phi_{S}^{\tau, t_{n-1}}\left(u^{n-1}\right), \quad n \geq 1, \quad \Phi_{S}^{\tau, t^{\prime}}(\eta):=\varphi_{T}^{\tau / 2} \circ \varphi_{V}^{\tau, t^{\prime}} \circ \varphi_{T}^{\tau / 2}(\eta) .
$$

We assume that $\int_{0}^{s} V^{\varepsilon}\left(\mathbf{x}, t^{\prime}+y\right) d y$ can be evaluated exactly in (2.3) and (2.4). For $m \in \mathbb{R}$, we denote by $\|\cdot\|_{m}$ the standard $H^{m}=H^{m}\left(\mathbb{T}^{d}\right)$ Sobolev norm, which reads as

$$
\|u\|_{m}^{2}=\sum_{k \in \mathbb{Z}^{d}}\left(1+|k|^{2}\right)^{m}\left|\widehat{u}_{k}\right|^{2}, \quad \text { where } \quad \widehat{u}_{k}=\frac{1}{(2 \pi)^{d}} \int_{\mathbb{T}^{d}} u(\mathbf{x}) e^{-i k \mathbf{x}} d \mathbf{x} .
$$

For $m=0$, the space is exactly $L^{2}$ and the corresponding norm is denoted as $\|\cdot\|$ for simplicity. Throughout the paper we assume $\sigma>d / 2$ so that the well-known bilinear estimate holds [1]:

$$
\|f g\|_{\sigma} \leq C_{\sigma, d}\|f\|_{\sigma}\|g\|_{\sigma},
$$

where $C_{\sigma, d}$ represents a positive constant depending on $\sigma$ and $d$. We consider the problem (1.2) till some time $0<T_{0}<T^{*}$, where $T^{*}>0$ denotes the maximum existence time of the solution. We give the error bounds of the splitting schemes for solving (1.2) till $t=T_{0}$ without discretizing the space variable, while the full discretization case can be analyzed similarly. In practice, the spatial discretization can be done by either Fourier pseudo-spectral method [25] or Hermite collocation method $[17,23]$ regarding the consistent boundary conditions. As we are considering the periodic setup in (1.2), the Fourier pseudo-spectral method can be easily applied. Throughout the rest of the paper, to simplify the notations and simultaneously address the dependence of the error on $\varepsilon$, we adopt the notation $A \lesssim B$ to represent that there exists a generic constant $C>0$, which is independent of the time step $\tau$ (or $n$ ) and the parameter $\varepsilon$, such that $|A| \leq C B$.

2.2. Main results. For the Lie-Trotter splitting scheme (2.3), assume that the potential and the solution of the NLSE-OP (1.2) satisfy

$$
\begin{aligned}
& V^{\varepsilon} \in C\left(\left[0, T_{0}\right] ; H^{\sigma+2}\right), \quad u \in C\left(\left[0, T_{0}\right] ; H^{\sigma+2}\right) \cap C^{1}\left(\left[0, T_{0}\right] ; H^{\sigma}\right), \\
& \left\|V^{\varepsilon}\right\|_{L^{\infty}\left(\left[0, T_{0}\right] ; H^{\sigma+2}\right)}+\|u\|_{L^{\infty}\left([0, T] ; H^{\sigma+2}\right)}+\left\|\partial_{t} u\right\|_{L^{\infty}\left([0, T] ; H^{\sigma}\right)} \lesssim 1,
\end{aligned}
$$

where $\sigma>d / 2$ is a real number. Then we have the following error estimate which shows uniform accuracy of the Lie-Trotter splitting scheme.

Theorem 2.1. (Lie-Trotter) Under the regularity assumption (2.6), there exists $\tau_{0}>0$ independent of $\varepsilon$ and $\tau$ (or $n)$, such that when $0<\tau \leq \tau_{0}$, the error of the Lie-Trotter scheme satisfies:

$$
\left\|u^{n}-u\left(\cdot, t_{n}\right)\right\|_{\sigma} \lesssim \tau, \quad 0 \leq n \leq T_{0} / \tau .
$$

For the Strang splitting scheme, assume that the given potential and the solution of the NLSE-OP (1.2) satisfy

$$
\begin{aligned}
& V^{\varepsilon} \in C\left(\left[0, T_{0}\right] ; H^{\sigma+4}\right) \cap C^{1}\left(\left[0, T_{0}\right] ; H^{\sigma+2}\right), \\
& u \in C\left(\left[0, T_{0}\right] ; H^{\sigma+4}\right) \cap C^{1}\left(\left[0, T_{0}\right] ; H^{\sigma+2}\right) \cap C^{2}\left(\left[0, T_{0}\right] ; H^{\sigma}\right), \\
& \left\|V^{\varepsilon}\right\|_{L^{\infty}\left(\left[0, T_{0}\right] ; H^{\sigma+4}\right)}+\varepsilon\left\|\partial_{t} V^{\varepsilon}\right\|_{L^{\infty}\left(\left[0, T_{0}\right] ; H^{\sigma+2}\right)} \lesssim 1 \\
& \|u\|_{L^{\infty}\left(\left[0, T_{0}\right] ; H^{\sigma+4}\right)}+\left\|\partial_{t} u\right\|_{L^{\infty}\left(\left[0, T_{0}\right] ; H^{\sigma+2}\right)}+\varepsilon\left\|\partial_{t t} u\right\|_{L^{\infty}\left(\left[0, T_{0}\right] ; H^{\sigma}\right)} \lesssim 1,
\end{aligned}
$$


with $\sigma>d / 2$. The error bound of the Strang splitting scheme is stated as the follows.

Theorem 2.2. (Strang) Under the regularity assumption (2.7), there exists $\tau_{0}>0$ independent of $\varepsilon$ and $\tau$ (or $n$ ), such that when $0<\tau \leq \tau_{0}$, the following error estimate of the Strang splitting scheme holds:

$$
\left\|u^{n}-u\left(\cdot, t_{n}\right)\right\|_{\sigma} \lesssim \min \left\{\tau, \frac{\tau^{2}}{\varepsilon}\right\}, \quad 0 \leq n \leq T_{0} / \tau .
$$

2.3. Extension to Klein-Gordon equation. The splitting schemes for the NLSE-OP (1.2) and corresponding error estimates can be extended to other wave or dispersive equations with a highly oscillatory potential. In the following, we present the extension to the nonlinear Klein-Gordon equation.

For describing cosmic superfluid, the rotating nonlinear Klein-Gordon equation was introduced, see e.g. [27] and the references therein. After applying the rotating Lagrangian coordinates transform, the following nonlinear Klein-Gordon equation with a highly oscillatory potential (KGE-OP) occurs [22]:

$$
\begin{aligned}
& \partial_{t t} u(\mathbf{x}, t)-\Delta u(\mathbf{x}, t)+u(\mathbf{x}, t)+V^{\varepsilon}(\mathbf{x}, t) u(\mathbf{x}, t)+f(u(\mathbf{x}, t))=0, \quad \mathbf{x} \in \mathbb{T}^{d}, \quad t>0, \\
& u(\mathbf{x}, 0)=u_{0}(\mathbf{x}), \quad \partial_{t} u(\mathbf{x}, 0)=u_{1}(\mathbf{x}), \quad \mathbf{x} \in \mathbb{T}^{d},
\end{aligned}
$$

where $u$ is the unknown, $u_{0}$ and $u_{1}$ are given real-valued initial data, $f: \mathbb{R} \rightarrow \mathbb{R}$ is the given nonlinearity and $V^{\varepsilon}$ is the highly oscillatory potential. In [22], the highly oscillatory $V^{\varepsilon}$ is an anisotropic trapping potential that composites with rapid rotation. The KGE-OP (2.8) also shows up in the counterpart of the study for the subsonic limit of the Klein-Gordon-Zakharov system [4, 20]. To apply the splitting schemes, we firstly rewrite (2.8) into a first order system by introducing

$$
v(\mathbf{x}, t)=\langle\nabla\rangle^{-1} \partial_{t} u(\mathbf{x}, t), \quad\langle\nabla\rangle=\sqrt{1-\Delta} .
$$

Then the vectorised equation reads

$$
\partial_{t}\left(\begin{array}{l}
u \\
v
\end{array}\right)=\left(\begin{array}{cc}
0 & \langle\nabla\rangle \\
-\langle\nabla\rangle & 0
\end{array}\right)\left(\begin{array}{l}
u \\
v
\end{array}\right)-\left(\begin{array}{c}
0 \\
\langle\nabla\rangle^{-1}\left(V^{\varepsilon} u+f(u)\right)
\end{array}\right), \quad t>0
$$

Note that the linear operator

$$
\left(\begin{array}{cc}
0 & \langle\nabla\rangle \\
-\langle\nabla\rangle & 0
\end{array}\right)
$$

is skew symmetric and $0<\langle\nabla\rangle^{-1} \leq 1$, then (2.9) is analogous to the NLSE-OP (1.2). One can thus split (2.9) into

$$
\partial_{t}\left(\begin{array}{l}
u_{1} \\
v_{1}
\end{array}\right)=\left(\begin{array}{cc}
0 & \langle\nabla\rangle \\
-\langle\nabla\rangle & 0
\end{array}\right)\left(\begin{array}{l}
u_{1} \\
v_{1}
\end{array}\right) \quad \text { and } \quad \partial_{t}\left(\begin{array}{l}
u_{2} \\
v_{2}
\end{array}\right)=-\left(\begin{array}{c}
0 \\
\langle\nabla\rangle^{-1}\left(V^{\varepsilon} u_{2}+f\left(u_{2}\right)\right)
\end{array}\right) .
$$

The subproblems can be exactly integrated respectively as

$$
\left(\begin{array}{c}
u_{1}(\mathbf{x}, t) \\
v_{1}(\mathbf{x}, t)
\end{array}\right)=\left(\begin{array}{cc}
\cos (\langle\nabla\rangle t) & \sin (\langle\nabla\rangle t) \\
-\sin (\langle\nabla\rangle t) & \cos (\langle\nabla\rangle t)
\end{array}\right)\left(\begin{array}{c}
u_{1}(\mathbf{x}, 0) \\
v_{1}(\mathbf{x}, 0)
\end{array}\right)
$$

and

$$
\left(\begin{array}{l}
u_{2}(\mathbf{x}, t) \\
v_{2}(\mathbf{x}, t)
\end{array}\right)=\left(\begin{array}{c}
u_{2}(\mathbf{x}, 0) \\
v_{2}(\mathbf{x}, 0)-\langle\nabla\rangle^{-1}\left[\int_{0}^{t} V^{\varepsilon}(\mathbf{x}, s) \mathrm{d} s u_{2}(\mathbf{x}, 0)+t f\left(u_{2}(\mathbf{x}, 0)\right)\right]
\end{array}\right) .
$$

It is straightforward to apply the Lie-Trotter or Strang splitting scheme, and the theoretical error estimates hold as well. Compared to the strategy introduced in [6] that integrates the oscillation in phase space with convolution, the splitting schemes could surely improve the efficiency. 


\section{ERror estimate of the Lie-Trotter Splitting}

In this section, we aim to prove Theorem 2.1 for the error bound of the Lie-Trotter splitting scheme (2.3) for solving the NLSE-OP (1.2). Denote

$$
R=\|u\|_{L^{\infty}\left([0, T] ; H^{\sigma}\right)}, \quad B_{R}^{\sigma}=\left\{v \in H^{\sigma}:\|v\|_{\sigma} \leq R\right\} .
$$

Firstly we introduce some auxiliary results which will be used in our proof.

Proposition 3.1. [13] For any function $g \in C^{\infty}(\mathbb{C}, \mathbb{C})$, there exists a nondecreasing function $\chi_{g}$ : $\mathbb{R}^{+} \rightarrow \mathbb{R}^{+}$such that

$$
\|g(u)\|_{\sigma} \leq\|g(0)\|_{\sigma}+\chi_{g}\left(\|u\|_{L^{\infty}}\right)\|u\|_{\sigma}, \quad \forall u \in H^{\sigma} .
$$

For all $v, w \in B_{R}^{\sigma}$, we have

$$
\|g(v)-g(w)\|_{\sigma} \leq \alpha(g, R)\|v-w\|_{\sigma},
$$

where $\alpha(g, R)=\left\|g^{\prime}(0)\right\|_{\sigma}+R \chi_{g^{\prime}}(c R)$, with $c>0$ being the constant for the Sobolev imbedding $\|\cdot\|_{L^{\infty}} \leq c\|\cdot\|_{\sigma}$.

Applying the triangle inequality, (3.1) and (3.2), we have

$$
\left\|f\left(|v|^{2}\right) v-f\left(|w|^{2}\right) w\right\|_{\sigma} \leq M_{0}\|v-w\|_{\sigma}, \quad v, w \in B_{R}^{\sigma},
$$

with $M_{0}=C_{\sigma, d}\|f(0)\|_{\sigma}+C_{\sigma, d}^{2} R^{2}\left[\chi_{f}\left(c^{2} R^{2}\right)+2 \alpha\left(f, C_{\sigma, d} R^{2}\right)\right]$.

Next we establish the stability result and the local truncation error.

Lemma 3.2. (Stability) For $v, w \in B_{R}^{\sigma}$ and $0 \leq t^{\prime} \leq T_{0}-\tau$, the propagator of the Lie-Trotter splitting scheme (2.3) satisfies:

$$
\left\|\Phi_{L}^{\tau, t^{\prime}}(v)-\Phi_{L}^{\tau, t^{\prime}}(w)\right\|_{\sigma} \leq \mathrm{e}^{M \tau}\|v-w\|_{\sigma}
$$

where $M>0$ depends on $\sigma, d, R,\left\|V^{\varepsilon}\right\|_{L^{\infty}\left(\left[0, T_{0}\right] ; H^{\sigma}\right)}$ and $f$.

Proof. Noticing $\varphi_{T}^{\tau}$ preserves $H^{\sigma}$-norm, we get

$$
\left\|\Phi_{L}^{\tau, t^{\prime}}(v)-\Phi_{L}^{\tau, t^{\prime}}(w)\right\|_{\sigma}=\left\|\varphi_{V}^{\tau, t^{\prime}}(v)-\varphi_{V}^{\tau, t^{\prime}}(w)\right\|_{\sigma} .
$$

Denote $\tilde{v}(\mathbf{x}, t)=\varphi_{V}^{t, t^{\prime}}(v)$ and $\tilde{w}(\mathbf{x}, t)=\varphi_{V}^{t, t^{\prime}}(w)$. Define $g(x):=\mathrm{e}^{-i x}$. It is obvious that $g \in$ $C^{\infty}(\mathbb{C}, \mathbb{C})$. It follows from $(3.1)$ that

$$
\begin{aligned}
\left\|\mathrm{e}^{-i \int_{0}^{t} V^{\varepsilon}\left(t^{\prime}+s\right) \mathrm{d} s}\right\|_{\sigma} & \leq\|g(0)\|_{\sigma}+\chi_{g}\left(\left\|\int_{0}^{t} V^{\varepsilon}\left(t^{\prime}+s\right) d s\right\|_{L^{\infty}}\right)\left\|\int_{0}^{t} V^{\varepsilon}\left(t^{\prime}+s\right) d s\right\|_{\sigma} \\
& \leq\|I d\|_{\sigma}+\chi_{g}\left(c t \sup _{0 \leq s \leq t}\left\|V^{\varepsilon}\left(t^{\prime}+s\right)\right\|_{\sigma}\right) t \sup _{0 \leq s \leq t}\left\|V^{\varepsilon}\left(t^{\prime}+s\right)\right\|_{\sigma} .
\end{aligned}
$$

Similarly, we get

$$
\begin{aligned}
\left\|\mathrm{e}^{-i t f\left(|v|^{2}\right)}\right\|_{\sigma} & \leq\|I d\|_{\sigma}+t \chi_{g}\left(t\|f\|_{L^{\infty}}\right)\left\|f\left(|v|^{2}\right)\right\|_{\sigma} \\
& \leq\|I d\|_{\sigma}+t \chi_{g}\left(t\|f\|_{L^{\infty}}\right)\left[\|f(0)\|_{\sigma}+C_{\sigma, d} \chi_{f}\left(c^{2}\|v\|_{\sigma}^{2}\right)\|v\|_{\sigma}^{2}\right] .
\end{aligned}
$$

Combining the above inequalities and using the bilinear inequality (2.5), we obtain

$$
\|\tilde{v}(\mathbf{x}, t)\|_{\sigma}=\left\|\varphi_{V}^{t, t^{\prime}}(v)\right\|_{\sigma} \leq C_{\sigma, d}^{2}\|v\|_{\sigma}\left\|\mathrm{e}^{-i \int_{0}^{t} V^{\varepsilon}\left(t^{\prime}+s\right) \mathrm{d} s}\right\|_{\sigma}\left\|\mathrm{e}^{-i t f\left(|v|^{2}\right)}\right\|_{\sigma} \leq M_{1}\|v\|_{\sigma},
$$

where $M_{1}$ depends on $\sigma, d, t, f,\|v\|_{\sigma}$ and $\sup _{0 \leq s \leq t}\left\|V^{\varepsilon}\left(t^{\prime}+s\right)\right\|_{\sigma}$. This implies that $\tilde{v}(\mathbf{x}, t), \tilde{w}(\mathbf{x}, t) \in H^{\sigma}$.

By Duhamel's formula, Minkovski's inequality, (3.3) and the bilinear inequality (2.5), we have

$$
\begin{aligned}
\|\tilde{v}(\cdot, \tau)-\tilde{w}(\cdot, \tau)\|_{\sigma} \leq & \|v-w\|_{\sigma}+\int_{0}^{\tau}\left\|V^{\varepsilon}\left(\cdot, t+t^{\prime}\right)(\tilde{v}(\cdot, t)-\tilde{w}(\cdot, t))\right\|_{\sigma} \mathrm{d} t \\
& +\int_{0}^{\tau}\left\|f\left(|\tilde{v}(\cdot, t)|^{2}\right) \tilde{v}(\mathbf{x}, t)-f\left(|\tilde{w}(\cdot, t)|^{2}\right) \tilde{w}(\cdot, t)\right\|_{\sigma} d t \\
& \leq\|v-w\|_{\sigma}+\left(M_{0}+C_{\sigma, d} \sup _{t \in\left[0, T_{0}\right]}\left\|V^{\varepsilon}\right\|_{\sigma}\right) \int_{0}^{\tau}\|\tilde{v}(\cdot, t)-\tilde{w}(\cdot, t)\|_{\sigma} \mathrm{d} t
\end{aligned}
$$


By Gronwall's inequality, we get

$$
\|\tilde{v}(\cdot, \tau)-\tilde{w}(\cdot, \tau)\|_{\sigma} \leq \mathrm{e}^{M \tau}\|v-w\|_{\sigma},
$$

where $M=M_{0}+C_{\sigma, d}\left\|V^{\varepsilon}\right\|_{L^{\infty}\left(\left[0, T_{0}\right] ; H^{\sigma}\right)}$ depends on $\sigma, d, R,\left\|V^{\varepsilon}\right\|_{L^{\infty}\left(\left[0, T_{0}\right] ; H^{\sigma}\right)}$ and $f$.

Omit the space variable for simplicity and denote the local truncation error for $n \geq 0$ as

$$
\xi^{n}:=u\left(t_{n+1}\right)-\Phi_{L}^{\tau, t_{n}}\left(u\left(t_{n}\right)\right)=u\left(t_{n+1}\right)-\mathrm{e}^{-i \tau \Delta}\left(\mathrm{e}^{-i \int_{0}^{\tau} V^{\varepsilon}\left(s+t_{n}\right) \mathrm{d} s-i \tau f\left(\left|u\left(t_{n}\right)\right|^{2}\right)} u\left(t_{n}\right)\right) .
$$

For the local truncation error, we have the following estimate.

Lemma 3.3. (Local error) Under the regularity assumption (2.6), the local truncation error of the Lie-Trotter splitting scheme (2.3) satisfies

$$
\left\|\xi^{n}\right\|_{\sigma} \lesssim \tau^{2}, \quad 0 \leq n<T_{0} / \tau
$$

Proof. For simplicity of notation, we denote $F_{n}(s)=f\left(\left|u\left(t_{n}+s\right)\right|^{2}\right), V_{n}^{\varepsilon}(s)=V^{\varepsilon}\left(t_{n}+s\right)$. By Taylor expansion, we have

$$
\begin{aligned}
\xi^{n} & =u\left(t_{n+1}\right)-\mathrm{e}^{-i \tau \Delta}\left[u\left(t_{n}\right)-i \int_{0}^{\tau} V_{n}^{\varepsilon}(s) \mathrm{d} s u\left(t_{n}\right)-i \tau f\left(\left|u\left(t_{n}\right)\right|^{2}\right) u\left(t_{n}\right)\right. \\
& \left.-\int_{0}^{1}(1-\theta) \mathrm{e}^{-i \theta \int_{0}^{\tau} V_{n}^{\varepsilon}(s) \mathrm{d} s-i \theta \tau f\left(\left|u\left(t_{n}\right)\right|^{2}\right)} \mathrm{d} \theta\left(\int_{0}^{\tau} V_{n}^{\varepsilon}(s) \mathrm{d} s+\tau f\left(\left|u\left(t_{n}\right)\right|^{2}\right)\right)^{2} u\left(t_{n}\right)\right] .
\end{aligned}
$$

Duhamel's principle gives that

$$
\begin{aligned}
u\left(t_{n+1}\right)= & \mathrm{e}^{-i \tau \Delta} u\left(t_{n}\right)+\int_{0}^{\tau} \mathrm{e}^{-i(\tau-s) \Delta}\left[-i V_{n}^{\varepsilon}(s) u\left(t_{n}+s\right)-i F_{n}(s) u\left(t_{n}+s\right)\right] \mathrm{d} s \\
= & \mathrm{e}^{-i \tau \Delta} u\left(t_{n}\right)-i \mathrm{e}^{-i \tau \Delta} \int_{0}^{\tau}\left[V_{n}^{\varepsilon}(s) u\left(t_{n}\right)+f\left(\left|u\left(t_{n}\right)\right|^{2}\right) u\left(t_{n}\right)\right] \mathrm{d} s \\
& +\mathrm{e}^{-i \tau \Delta} \int_{0}^{\tau} \int_{0}^{1} \mathrm{e}^{i s \theta \Delta} \mathrm{d} \theta(s \Delta)\left[V_{n}^{\varepsilon}(s) u\left(t_{n}+s\right)+F_{n}(s) u\left(t_{n}+s\right)\right] \mathrm{d} s \\
& -i \mathrm{e}^{-i \tau \Delta} \int_{0}^{\tau}\left[V_{n}^{\varepsilon}(s)\left(u\left(t_{n}+s\right)-u\left(t_{n}\right)\right)+F_{n}(s) u\left(t_{n}+s\right)-F_{n}(0) u\left(t_{n}\right)\right] \mathrm{d} s .
\end{aligned}
$$

Thus the local error can be written as

$$
\begin{aligned}
\xi^{n}= & \mathrm{e}^{-i \tau \Delta}\left[\int_{0}^{1}(1-\theta) \mathrm{e}^{-i \theta \int_{0}^{\tau} V_{n}^{\varepsilon}(s) \mathrm{d} s-i \theta \tau f\left(\left|u\left(t_{n}\right)\right|^{2}\right)} \mathrm{d} \theta\left(\int_{0}^{\tau} V_{n}^{\varepsilon}(s) \mathrm{d} s+\tau f\left(\left|u\left(t_{n}\right)\right|^{2}\right)\right)^{2} u\left(t_{n}\right)\right] \\
& +\mathrm{e}^{-i \tau \Delta} \int_{0}^{\tau} \int_{0}^{1} \mathrm{e}^{i s \theta \Delta} d \theta(s \Delta)\left[V_{n}^{\varepsilon}(s) u\left(t_{n}+s\right)+F_{n}(s) u\left(t_{n}+s\right)\right] \mathrm{d} s \\
& -i \mathrm{e}^{-i \tau \Delta} \int_{0}^{\tau}\left[V_{n}^{\varepsilon}(s)\left(u\left(t_{n}+s\right)-u\left(t_{n}\right)\right)+F_{n}(s) u\left(t_{n}+s\right)-F_{n}(0) u\left(t_{n}\right)\right] \mathrm{d} s
\end{aligned}
$$

By using (2.5) and (3.2), we arrive at

$$
\begin{aligned}
\left\|\xi^{n}\right\|_{\sigma} \leq & \tau^{2} C_{\sigma, d}^{3}\left\|u\left(t_{n}\right)\right\|_{\sigma}\left[\left\|f\left(\left|u\left(t_{n}\right)\right|^{2}\right)\right\|_{\sigma}^{2}+\sup _{0 \leq s \leq \tau}\left\|V_{n}^{\varepsilon}(s)\right\|_{\sigma}\right] \sup _{0 \leq \theta \leq 1}\left\|\mathrm{e}^{-i \theta \int_{0}^{\tau} V_{n}^{\varepsilon}(s) \mathrm{d} s-i \theta \tau f\left(\left|u\left(t_{n}\right)\right|^{2}\right)}\right\|_{\sigma} \\
& +\tau^{2} C_{\sigma+2, d} \sup _{0 \leq s \leq \tau}\left\|u\left(t_{n}+s\right)\right\|_{\sigma+2}\left(\left\|V_{n}^{\varepsilon}(s)\right\|_{\sigma+2}+\left\|F_{n}(s)\right\|_{\sigma+2}\right) \\
& +\left(M_{0}+\sup _{0 \leq s \leq \tau}\left\|V_{n}^{\varepsilon}(s)\right\|_{\sigma}\right) \int_{0}^{\tau}\left\|u\left(t_{n}+s\right)-u\left(t_{n}\right)\right\|_{\sigma} d s .
\end{aligned}
$$

Applying similar arguments as the proof of Lemma 3.2, we have

$$
\left\|\mathrm{e}^{-i \theta \int_{0}^{\tau} V_{n}^{\varepsilon}(s) \mathrm{d} s-i \theta \tau f\left(\left|u\left(t_{n}\right)\right|^{2}\right)}\right\|_{\sigma} \leq M_{2},
$$

where $M_{2}$ depends on $\sigma, d, \tau, f,\left\|u\left(t_{n}\right)\right\|_{\sigma}$ and $\sup _{0 \leq s \leq \tau}\left\|V_{n}^{\varepsilon}(s)\right\|_{\sigma}$. Owing to (3.1), we obtain

$$
\left\|F_{n}(s)\right\|_{\sigma+2} \leq\|f(0)\|_{\sigma+2}+C_{\sigma+2, d} \chi_{f}\left(c^{2}\left\|u\left(t_{n}+s\right)\right\|_{\sigma}^{2}\right)\left\|u\left(t_{n}+s\right)\right\|_{\sigma+2}^{2},
$$


and

$$
\begin{aligned}
\int_{0}^{\tau}\left\|u\left(t_{n}+s\right)-u\left(t_{n}\right)\right\|_{\sigma} d s & =\int_{0}^{\tau}\left\|\int_{0}^{s} \partial_{t} u\left(t_{n}+y\right) d y\right\|_{\sigma} d s \\
& \leq \int_{0}^{\tau} \int_{0}^{s}\left\|\partial_{t} u\left(t_{n}+y\right)\right\|_{\sigma} d y d s \leq \tau^{2} \sup _{0 \leq s \leq \tau}\left\|\partial_{t} u\left(t_{n}+s\right)\right\|_{\sigma} .
\end{aligned}
$$

Hence we conclude that there exists a constant $C>0$ such that

$$
\left\|\xi^{n}\right\|_{\sigma} \leq C \tau^{2}, \quad 0 \leq n<T_{0} / \tau
$$

where $C$ depends on $\sigma, d, f,\left\|V^{\varepsilon}\right\|_{L^{\infty}\left(\left[0, T_{0}\right] ; H^{\sigma+2}\right)},\|u\|_{L^{\infty}\left(\left[0, T_{0}\right] ; H^{\sigma+2}\right)}$ and $\left\|\partial_{t} u\right\|_{L^{\infty}\left(\left[0, T_{0}\right] ; H^{\sigma}\right)}$.

With the above two lemmas, we give the proof of the global error estimate for the Lie-Trotter splitting (2.3) which is stated as Theorem 2.1.

Proof of Theorem 2.1.

Proof. We use an induction argument for the boundedness of the numerical solution. Denote $R=$ $\|u\|_{L^{\infty}\left(\left[0, T_{0}\right] ; H^{\sigma}\right)}$. We next show the numerical solution $u^{n} \in B_{R+1}^{\sigma}$. Firstly, it is obvious for $n=0$ since $u^{0}=u_{0} \in B_{R}^{\sigma}$. Assume $u^{l} \in B_{R+1}^{\sigma-2}$ for $0 \leq l \leq n<T_{0} / \tau$. Denote $e^{n}=u\left(t_{n}\right)-u^{n}$. Taking the difference between (2.3) and (3.5), we get

$$
e^{n+1}=\xi^{n}+\Phi_{L}^{\tau, t_{n}}\left(u\left(t_{n}\right)\right)-\Phi_{L}^{\tau, t_{n}}\left(u^{n}\right) .
$$

Using Lemmas 3.2 and 3.3, we get

$$
\begin{aligned}
\left\|e^{n+1}\right\|_{\sigma} & \leq\left\|\xi^{n}\right\|_{\sigma}+\left\|\Phi_{L}^{\tau, t_{n}}\left(u\left(t_{n}\right)\right)-\Phi_{L}^{\tau, t_{n}}\left(u^{n}\right)\right\|_{\sigma} \\
& \leq \mathrm{e}^{M \tau}\left\|e^{n}\right\|_{\sigma}+C \tau^{2} \leq \mathrm{e}^{M(n+1) \tau}\left\|e^{0}\right\|_{\sigma}+C \tau^{2} \sum_{l=0}^{n} \mathrm{e}^{M l \tau} .
\end{aligned}
$$

Then we get

$$
\left\|e^{n+1}\right\|_{\sigma} \leq C \frac{\mathrm{e}^{M T_{0}}-1}{M} \tau, \quad 0 \leq n<T_{0} / \tau
$$

and when $0<\tau \leq \tau_{0}:=\frac{M}{C\left(\mathrm{e}^{M T_{0}}-1\right)}$, we have

$$
\left\|u^{n+1}\right\|_{\sigma} \leq\left\|u\left(t_{n+1}\right)\right\|_{\sigma}+1 .
$$

Hence, we have $u^{n+1} \in B_{R+1}^{\sigma}$ and the induction proof is completed.

Remark 3.4. For the other Lie-Trotter splitting

$$
u^{n}=\prod_{m=1}^{n} \Psi_{L}^{\tau, t_{m-1}}\left(u_{0}\right), \quad \Psi_{L}^{\tau, t^{\prime}}(\xi):=\varphi_{V}^{\tau, t^{\prime}} \circ \varphi_{T}^{\tau}(\xi), \quad n \geq 0
$$

we can get the first-order convergence under the same regularity assumptions by noticing that

$$
\begin{aligned}
\left\|\Psi_{L}^{\tau, t^{\prime}}(v)-\Psi_{L}^{\tau, t^{\prime}}(w)\right\|_{\sigma} & \leq C_{\sigma, d}\left\|\mathrm{e}^{-i \int_{0}^{\tau} V^{\varepsilon}\left(t^{\prime}+y\right) d y}\right\|_{\sigma}\left\|\mathrm{e}^{-i \tau f\left(\left|e^{-i \tau \Delta} v\right|^{2}\right)} e^{-i \tau \Delta} v-\mathrm{e}^{-i \tau f\left(\left|e^{-i \tau \Delta} w\right|^{2}\right)} e^{-i \tau \Delta} w\right\|_{\sigma} \\
& \leq M\|v-w\|_{\sigma},
\end{aligned}
$$

where we have used (3.4) and (3.2).

\section{Error estamte of Strang splitting}

In this section, we establish the error bound of the Strang splitting scheme (2.4) by proving Theorem 2.2. We adopt the same notations as in the previous section.

Lemma 4.1. (Stability) For $v, w \in B_{R}^{\sigma}$ and $0 \leq t^{\prime} \leq T_{0}-\tau$, the propagator of the Strang splitting scheme (2.4) satisfies:

$$
\left\|\Phi_{S}^{\tau, t^{\prime}}(v)-\Phi_{S}^{\tau, t^{\prime}}(w)\right\|_{\sigma} \leq \mathrm{e}^{M \tau}\|v-w\|_{\sigma},
$$

for some $M>0$ depending on $\sigma, d, R,\left\|V^{\varepsilon}\right\|_{L^{\infty}\left(\left[0, T_{0}\right] ; H^{\sigma}\right)}$ and $f$. 
Proof. Directly by the fact that $\varphi_{T}^{\tau}$ preserves the $H^{s}$-norm and Lemma 3.2, we have

$$
\begin{aligned}
\left\|\Phi_{S}^{\tau, t^{\prime}}(v)-\Phi_{S}^{\tau, t^{\prime}}(w)\right\|_{\sigma} & =\left\|\varphi_{V}^{\tau, t^{\prime}} \circ \varphi_{T}^{\tau / 2}(v)-\varphi_{V}^{\tau, t^{\prime}} \circ \varphi_{T}^{\tau / 2}(w)\right\|_{H^{\sigma-4}} \\
& \leq \mathrm{e}^{M \tau}\left\|\varphi_{T}^{\tau / 2}(v)-\varphi_{T}^{\tau / 2}(w)\right\|_{\sigma}=\mathrm{e}^{M \tau}\|v-w\|_{\sigma}
\end{aligned}
$$

which completes the proof.

Denote the local truncation error of the Strang splitting for $n \geq 0$ as

$$
\eta^{n}:=u\left(t_{n+1}\right)-\Phi_{S}^{\tau, t_{n}}\left(u\left(t_{n}\right)\right)=u\left(t_{n+1}\right)-\mathrm{e}^{-i \frac{\tau}{2} \Delta}\left(\mathrm{e}^{-i \int_{0}^{\tau} V_{n}^{\varepsilon}(s) \mathrm{d} s-i \tau f\left(\left|\mathrm{e}^{-i \frac{\tau}{2} \Delta} u\left(t_{n}\right)\right|^{2}\right)} \mathrm{e}^{-i \frac{\tau}{2} \Delta} u\left(t_{n}\right)\right) .
$$

To do so, we further introduce some notations. Let $R=R(v, t, s)$ be a term that depends on the function values $v\left(t+t^{\prime}\right)$ for $0 \leq t^{\prime} \leq s$. We say that $R \in \mathcal{R}_{\beta}\left(v, s^{\alpha}\right)$ if and only if

$$
\|R(v, t, s)\|_{\sigma} \leq C s^{\alpha},
$$

where $C$ depends on $\sup _{0 \leq t^{\prime} \leq s}\left\|v\left(t+t^{\prime}\right)\right\|_{\sigma+\beta}$. Similarly, we denote by $R=R(v, w, t, s) \in \mathcal{R}_{\beta, \gamma}\left(v, w, s^{\alpha}\right)$ if and only if

$$
\|R(v, w, t, s)\|_{\sigma} \leq C s^{\alpha},
$$

where $C$ depends on $\sup _{0 \leq t^{\prime} \leq s}\left\|v\left(t+t^{\prime}\right)\right\|_{\sigma+\beta}$ and $\sup _{0 \leq t^{\prime} \leq s}\left\|w\left(t+t^{\prime}\right)\right\|_{\sigma+\gamma}$. For simplicity, we write $\phi=$ $\psi+\mathcal{R}_{\beta}\left(v, s^{\alpha}\right)$ whenever $\phi=\psi+R$ with $R \in \mathcal{R}_{\beta}\left(v, s^{\alpha}\right)$ and similarly $\phi=\psi+\mathcal{R}_{\beta, \gamma}\left(v, w, s^{\alpha}\right)$ whenever $\phi=\psi+R$ with $R \in \mathcal{R}_{\beta, \gamma}\left(v, w, s^{\alpha}\right)$. Next we introduce a lemma on expansion which will be used frequently afterwards.

Lemma 4.2. For all $s \geq 0$, we have

$$
\left\|e^{ \pm i s \Delta} v-v\right\|_{\sigma} \leq s\|v\|_{\sigma+2} \quad \text { and } \quad\left\|e^{ \pm i s \Delta} v-v \mp i s \Delta v\right\|_{\sigma} \leq \frac{s^{2}}{2}\|v\|_{\sigma+4} .
$$

Proof. For $v=\sum_{k \in \mathbb{Z}^{d}} \widehat{v}_{k} e^{i k \mathbf{x}}$, we have

$$
e^{ \pm i s \Delta} v-v=\sum_{k \in \mathbb{Z}^{d}} \widehat{v}_{k}\left(e^{\mp i s|k|^{2}}-1\right) e^{i k \mathbf{x}}
$$

which yields that

$$
\begin{aligned}
\left\|e^{ \pm i s \Delta} v-v\right\|_{\sigma}^{2} & =\sum_{k \in \mathbb{Z}^{d}}\left(1+|k|^{2}\right)^{\sigma}\left|\widehat{v}_{k}\right|^{2}\left|e^{\mp i s|k|^{2}}-1\right|^{2} \leq s^{2} \sum_{k \in \mathbb{Z}^{d}}\left(1+|k|^{2}\right)^{\sigma}\left|\widehat{v}_{k}\right|^{2}|k|^{4} \\
& \leq s^{2} \sum_{k \in \mathbb{Z}^{d}}\left(1+|k|^{2}\right)^{\sigma+2}\left|\widehat{v}_{k}\right|^{2} \leq s^{2}\|v\|_{\sigma+2}^{2} .
\end{aligned}
$$

Similarly, we have

$$
\begin{aligned}
\left\|e^{ \pm i s \Delta} v-v \mp i s \Delta v\right\|_{\sigma}^{2} & =\left.\left.\sum_{k \in \mathbb{Z}^{d}}\left(1+|k|^{2}\right)^{\sigma}\left|\widehat{v}_{k}\right|^{2}\left|e^{\mp i s|k|^{2}}-1 \pm i s\right| k\right|^{2}\right|^{2} \leq \frac{s^{4}}{4} \sum_{k \in \mathbb{Z}^{d}}\left(1+|k|^{2}\right)^{\sigma}\left|\widehat{v}_{k}\right|^{2}|k|^{8} \\
& \leq \frac{s^{4}}{4} \sum_{k \in \mathbb{Z}^{d}}\left(1+|k|^{2}\right)^{\sigma+4}\left|\widehat{v}_{k}\right|^{2} \leq \frac{s^{4}}{4}\|v\|_{\sigma+4}^{2}
\end{aligned}
$$

which completes the proof.

Lemma 4.3. (Local error) Under the regularity assumption (2.7), the local truncation error of the Strang splitting scheme (2.4) satisfies

$$
\left\|\xi^{n}\right\|_{\sigma} \lesssim \min \left\{\tau^{2}, \frac{\tau^{3}}{\varepsilon}\right\}, \quad 0 \leq n<T_{0} / \tau .
$$


Proof. Denote $u_{\frac{1}{2}}=\mathrm{e}^{-i \tau \Delta / 2} u\left(t_{n}\right)$. An application of the Duhamel's formula and Lemma 4.2 leads to the following representation for $0 \leq s \leq \tau$ :

$$
\begin{aligned}
u\left(t_{n}+s\right) & =\mathrm{e}^{-i s \Delta} u\left(t_{n}\right)-i \int_{0}^{s} \mathrm{e}^{-i(s-y) \Delta}\left[\left(V_{n}^{\varepsilon}(y)+F_{n}(y)\right) u\left(t_{n}+y\right)\right] d y \\
& =u_{\frac{1}{2}}+\left(\mathrm{e}^{-i(s-\tau / 2) \Delta}-1\right) u_{\frac{1}{2}}-i \int_{0}^{s} \mathrm{e}^{-i(s-y) \Delta}\left[\left(V_{n}^{\varepsilon}(y)+F_{n}(y)\right) u\left(t_{n}+y\right)\right] d y \\
& =u_{\frac{1}{2}}+\mathcal{R}_{2}(u, \tau)+\mathcal{R}_{0,0}\left(V_{n}^{\varepsilon}, u, \tau\right)+\mathcal{R}_{0,0}\left(F_{n}, u, \tau\right) \\
& =u_{\frac{1}{2}}+\mathcal{R}_{2}(u, \tau)+\mathcal{R}_{0,0}\left(V_{n}^{\varepsilon}, u, \tau\right),
\end{aligned}
$$

where we have used the inequality (3.1) that $\left\|F_{n}(y)\right\|_{\sigma} \leq\|f(0)\|_{\sigma}+C_{\sigma, d}\|u\|_{\sigma}^{2} \chi_{f}\left(c^{2}\|u\|_{\sigma}^{2}\right)$. Plugging this approximation into the Duhamel's formula and applying Lemma 4.2, we obtain

$$
\begin{aligned}
u\left(t_{n}+s\right) & =\mathrm{e}^{-i s \Delta} u\left(t_{n}\right)-i \int_{0}^{s} \mathrm{e}^{-i(s-y) \Delta}\left[\left(V_{n}^{\varepsilon}(y)+f\left(\left|u_{\frac{1}{2}}\right|^{2}\right)\right) u_{\frac{1}{2}}\right] d y+\mathcal{R}_{2}\left(u, \tau^{2}\right)+\mathcal{R}_{0,2}\left(V_{n}^{\varepsilon}, u, \tau^{2}\right) \\
& =\mathrm{e}^{i(\tau / 2-s) \Delta} u_{\frac{1}{2}}-i \int_{0}^{s} V_{n}^{\varepsilon}(y) u_{\frac{1}{2}} d y-i \int_{0}^{s} f\left(\left|u_{\frac{1}{2}}\right|^{2}\right) u_{\frac{1}{2}} d y+\mathcal{R}_{2}\left(u, \tau^{2}\right)+\mathcal{R}_{2,2}\left(V_{n}^{\varepsilon}, u, \tau^{2}\right) \\
& =\left[1+i\left(\frac{\tau}{2}-s\right) \Delta\right] u_{\frac{1}{2}}-i G_{n}^{\varepsilon}(s) u_{\frac{1}{2}}-i s f\left(\left|u_{\frac{1}{2}}\right|^{2}\right) u_{\frac{1}{2}}+\mathcal{R}_{4}\left(u, \tau^{2}\right)+\mathcal{R}_{2,2}\left(V_{n}^{\varepsilon}, u, \tau^{2}\right),
\end{aligned}
$$

where

$$
G_{n}^{\varepsilon}(s)=\int_{0}^{s} V_{n}^{\varepsilon}(y) d y .
$$

With this approximation, setting $s=\tau$ in the Duhamel's formula, one gets

$$
\begin{aligned}
u\left(t_{n}+\tau\right) & =\mathrm{e}^{-i \tau \Delta} u\left(t_{n}\right)-i \int_{0}^{\tau} \mathrm{e}^{-i(\tau-s) \Delta}\left[\left(V_{n}^{\varepsilon}(s)+F_{n}(s)\right) u\left(t_{n}+s\right)\right] \mathrm{d} s \\
& =\mathrm{e}^{-i \tau \Delta} u\left(t_{n}\right)-i \mathrm{e}^{-i \tau \Delta}\left(Q_{1}+Q_{2}\right)+\mathcal{R}_{0,4}\left(V_{n}^{\varepsilon}, u, \tau^{3}\right)+\mathcal{R}_{2,2}\left(V_{n}^{\varepsilon}, u, \tau^{3}\right),
\end{aligned}
$$

where

$$
\begin{aligned}
Q_{1} & =\int_{0}^{\tau} \mathrm{e}^{i s \Delta}\left[V_{n}^{\varepsilon}(s)\left(1+i\left(\frac{\tau}{2}-s\right) \Delta-i G_{n}^{\varepsilon}(s)-i s f\left(\left|u_{\frac{1}{2}}\right|^{2}\right)\right) u_{\frac{1}{2}}\right] \mathrm{d} s, \\
Q_{2} & =\int_{0}^{\tau} \mathrm{e}^{i s \Delta}\left[F_{n}(s) u\left(t_{n}+s\right)\right] \mathrm{d} s .
\end{aligned}
$$

Applying Lemma 4.2, $Q_{1}$ can be expanded as

$$
\begin{aligned}
Q_{1}= & \int_{0}^{\tau}(1+i s \Delta)\left(V_{n}^{\varepsilon}(s) u_{\frac{1}{2}}\right) \mathrm{d} s+i \int_{0}^{\tau} V_{n}^{\varepsilon}(s)\left[\left(\frac{\tau}{2}-s\right) \Delta-G_{n}^{\varepsilon}(s)-s f\left(\left|u_{\frac{1}{2}}\right|^{2}\right)\right] u_{\frac{1}{2}} \mathrm{~d} s+\mathcal{R}_{4,4}\left(V_{n}^{\varepsilon}, u, \tau^{3}\right) \\
= & G_{n}^{\varepsilon}(\tau) u_{\frac{1}{2}}+i \Delta\left(H_{n}^{\varepsilon}(\tau) u_{\frac{1}{2}}\right)+i\left[\frac{\tau}{2} G_{n}^{\varepsilon}(\tau)-H_{n}^{\varepsilon}(\tau)\right] \Delta u_{\frac{1}{2}} \\
& -i\left[\frac{G_{n}^{\varepsilon}(\tau)^{2}}{2}+H_{n}^{\varepsilon}(\tau) f\left(\left|u_{\frac{1}{2}}\right|^{2}\right)\right] u_{\frac{1}{2}}+\mathcal{R}_{4,4}\left(V_{n}^{\varepsilon}, u, \tau^{3}\right),
\end{aligned}
$$

where $H_{n}^{\varepsilon}(\tau)=\int_{0}^{\tau} s V_{n}^{\varepsilon}(s) d s$. Moreover, by Taylor expansion, we have

$$
\begin{aligned}
Q_{2} & =\int_{0}^{\tau} \mathrm{e}^{i s \Delta}\left[F_{n}\left(\frac{\tau}{2}\right) u\left(t_{n}+s\right)\right] \mathrm{d} s+\int_{0}^{\tau} \mathrm{e}^{i s \Delta}\left[\int_{\frac{\tau}{2}}^{s} F_{n}^{\prime}(y) d y u\left(t_{n}+s\right)\right] \mathrm{d} s \\
& =\int_{0}^{\tau} \mathrm{e}^{i s \Delta}\left[F_{n}\left(\frac{\tau}{2}\right) u\left(t_{n}+s\right)\right] \mathrm{d} s+\int_{0}^{\tau} \int_{\frac{\tau}{2}}^{s} F_{n}^{\prime}(y) d y u\left(t_{n}+s\right) \mathrm{d} s+\mathcal{R}_{2,2}\left(u, \partial_{t} u, \tau^{3}\right) \\
& =\int_{0}^{\tau} \mathrm{e}^{i s \Delta}\left[F_{n}\left(\frac{\tau}{2}\right) u\left(t_{n}+s\right)\right] \mathrm{d} s+\int_{0}^{\tau} \int_{\frac{\tau}{2}}^{s} F_{n}^{\prime}(y) d y u\left(t_{n}+\frac{\tau}{2}\right) \mathrm{d} s+\mathcal{R}_{2,2}\left(u, \partial_{t} u, \tau^{3}\right) \\
& =\int_{0}^{\tau} \mathrm{e}^{i s \Delta}\left[F_{n}\left(\frac{\tau}{2}\right) u\left(t_{n}+s\right)\right] \mathrm{d} s+u\left(t_{n}+\frac{\tau}{2}\right) \int_{0}^{\frac{\tau}{2}} y\left[F_{n}^{\prime}(\tau-y)-F_{n}^{\prime}(y)\right] d y+\mathcal{R}_{2,2}\left(u, \partial_{t} u, \tau^{3}\right) \\
& =\int_{0}^{\tau} \mathrm{e}^{i s \Delta}\left[F_{n}\left(\frac{\tau}{2}\right) u\left(t_{n}+s\right)\right] \mathrm{d} s+\mathcal{R}_{2,2}\left(u, \partial_{t} u, \tau^{3}\right)+\mathcal{R}_{0,0}\left(u, \partial_{t} u, \tau^{2}\right) .
\end{aligned}
$$


Noticing that

$$
\int_{0}^{\frac{\tau}{2}} y\left[F_{n}^{\prime}(\tau-y)-F_{n}^{\prime}(y)\right] d y=\int_{0}^{\frac{\tau}{2}} F_{n}^{\prime \prime}(s) \frac{s^{2}}{2} \mathrm{~d} s+\int_{\frac{\tau}{2}}^{\tau} F_{n}^{\prime \prime}(s) \frac{(\tau-s)^{2}}{2} \mathrm{~d} s
$$

this implies

$$
Q_{2}=\int_{0}^{\tau} \mathrm{e}^{i s \Delta}\left[F_{n}\left(\frac{\tau}{2}\right) u\left(t_{n}+s\right)\right] \mathrm{d} s+\mathcal{R}_{2,2}\left(u, \partial_{t} u, \tau^{3}\right)+\mathcal{R}_{0,0}\left(u, \partial_{t t} u, \tau^{3}\right),
$$

where we have used (3.1) repeatedly for $f, f^{\prime}$ and $f^{\prime \prime}$. Setting $s=\frac{\tau}{2}$ in (4.3), we have

$$
u\left(t_{n}+\frac{\tau}{2}\right)=u_{\frac{1}{2}}-i G_{n}^{\varepsilon}\left(\frac{\tau}{2}\right) u_{\frac{1}{2}}-\frac{i \tau}{2} f\left(\left|u_{\frac{1}{2}}\right|^{2}\right) u_{\frac{1}{2}}+\mathcal{R}_{4}\left(u, \tau^{2}\right)+\mathcal{R}_{2,2}\left(V_{n}^{\varepsilon}, u, \tau^{2}\right),
$$

which yields that

$$
\begin{aligned}
& \left|u\left(t_{n}+\frac{\tau}{2}\right)\right|^{2}=\left|u_{\frac{1}{2}}\right|^{2}+\mathcal{R}_{4}\left(u, \tau^{2}\right)+\mathcal{R}_{2,2}\left(V_{n}^{\varepsilon}, u, \tau^{2}\right), \\
& F_{n}\left(\frac{\tau}{2}\right)=f\left(\left|u_{\frac{1}{2}}\right|^{2}\right)+\mathcal{R}_{4}\left(u, \tau^{2}\right)+\mathcal{R}_{2,2}\left(V_{n}^{\varepsilon}, u, \tau^{2}\right) .
\end{aligned}
$$

Plugging this approximation and (4.3) into $Q_{2}$, using Lemma 4.2, we get

$$
\begin{aligned}
Q_{2} & =\int_{0}^{\tau} \mathrm{e}^{i s \Delta}\left[f\left(\left|u_{\frac{1}{2}}\right|^{2}\right) u\left(t_{n}+s\right)\right] \mathrm{d} s+Q_{3} \\
& =\int_{0}^{\tau} \mathrm{e}^{i s \Delta}\left[f\left(\left|u_{\frac{1}{2}}\right|^{2}\right)\left(1+i\left(\frac{\tau}{2}-s\right) \Delta-i G_{n}^{\varepsilon}(s)-i s f\left(\left|u_{\frac{1}{2}}\right|^{2}\right)\right) u_{\frac{1}{2}}\right] \mathrm{d} s+Q_{3} \\
& =\int_{0}^{\tau}(1+i s \Delta)\left(f\left(\left|u_{\frac{1}{2}}\right|^{2}\right) u_{\frac{1}{2}}\right) \mathrm{d} s+i f\left(\left|u_{\frac{1}{2}}\right|^{2}\right) \int_{0}^{\tau}\left[\left(\frac{\tau}{2}-s\right) \Delta-G_{n}^{\varepsilon}(s)-s f\left(\left|u_{\frac{1}{2}}\right|^{2}\right)\right] u_{\frac{1}{2}} \mathrm{~d} s+Q_{3} \\
& =\tau f\left(\left|u_{\frac{1}{2}}\right|^{2}\right) u_{\frac{1}{2}}+\frac{i \tau^{2}}{2} \Delta\left(f\left(\left|u_{\frac{1}{2}}\right|^{2}\right) u_{\frac{1}{2}}\right)-i \int_{0}^{\tau} G_{n}^{\varepsilon}(s) \mathrm{d} s f\left(\left|u_{\frac{1}{2}}\right|^{2}\right) u_{\frac{1}{2}}-\frac{i \tau^{2}}{2} f\left(\left|u_{\frac{1}{2}}\right|^{2}\right)^{2} u_{\frac{1}{2}}+Q_{3},
\end{aligned}
$$

where by (4.4) and (4.5),

$$
Q_{3}=\mathcal{R}_{4}\left(u, \tau^{3}\right)+\mathcal{R}_{2,2}\left(V_{n}^{\varepsilon}, u, \tau^{3}\right)+\mathcal{R}_{2,2}\left(u, \partial_{t} u, \tau^{3}\right)+\min \left\{\mathcal{R}_{0,0}\left(u, \partial_{t} u, \tau^{2}\right), \mathcal{R}_{0,0}\left(u, \partial_{t t} u, \tau^{3}\right)\right\}
$$

This concludes that

$$
\begin{aligned}
u\left(t_{n}+\tau\right)= & \mathrm{e}^{-i \tau \Delta} u\left(t_{n}\right)-i G_{n}^{\varepsilon}(\tau) u_{\frac{1}{2}}-\tau \Delta\left(G_{n}^{\varepsilon}(\tau) u_{\frac{1}{2}}\right)+\Delta\left(H_{n}^{\varepsilon}(\tau) u_{\frac{1}{2}}\right)+\left(\frac{\tau}{2} G_{n}^{\varepsilon}(\tau)-H_{n}^{\varepsilon}(\tau)\right) \Delta u_{\frac{1}{2}} \\
& -u_{\frac{1}{2}}\left[\frac{G_{n}^{\varepsilon}(\tau)^{2}}{2}+\left(i \tau+H_{n}^{\varepsilon}(\tau)\right) f\left(\left|u_{\frac{1}{2}}\right|^{2}\right)\right]-\frac{\tau^{2}}{2} \Delta\left(f\left(\left|u_{\frac{1}{2}}\right|^{2}\right) u_{\frac{1}{2}}\right)-\frac{\tau^{2}}{2} f\left(\left|u_{\frac{1}{2}}\right|^{2}\right)^{2} u_{\frac{1}{2}} \\
& -\int_{0}^{\tau} G_{n}^{\varepsilon}(s) \mathrm{d} s f\left(\left|u_{\frac{1}{2}}\right|^{2}\right) u_{\frac{1}{2}}+\mathcal{R}_{4,4}\left(V_{n}^{\varepsilon}, u, \tau^{3}\right)+\mathcal{R}_{2,2}\left(u, \partial_{t} u, \tau^{3}\right) \\
& +\min \left\{\mathcal{R}_{0,0}\left(u, \partial_{t} u, \tau^{2}\right), \mathcal{R}_{0,0}\left(u, \partial_{t t} u, \tau^{3}\right)\right\}
\end{aligned}
$$

On the other hand, an application of (4.2) yields

$$
\begin{aligned}
\Phi_{S}^{\tau, t_{n}}\left(u\left(t_{n}\right)\right)= & \mathrm{e}^{-i \tau \Delta / 2}\left(\mathrm{e}^{-i G_{n}^{\varepsilon}(\tau)-i \tau f\left(\left|u_{\frac{1}{2}}\right|^{2}\right)} u_{\frac{1}{2}}\right) \\
= & \mathrm{e}^{-i \tau \Delta / 2}\left(\left[1-i G_{n}^{\varepsilon}(\tau)-i \tau f\left(\left|u_{\frac{1}{2}}\right|^{2}\right)-\frac{1}{2}\left(G_{n}^{\varepsilon}(\tau)+\tau f\left(\left|u_{\frac{1}{2}}\right|^{2}\right)\right)^{2}\right] u_{\frac{1}{2}}\right)+\mathcal{R}_{0,0}\left(V_{n}^{\varepsilon}, u, \tau^{3}\right) \\
= & \mathrm{e}^{-i \tau \Delta} u\left(t_{n}\right)-i\left(G_{n}^{\varepsilon}(\tau)+\tau f\left(\left|u_{\frac{1}{2}}\right|^{2}\right)\right) u_{\frac{1}{2}}-\frac{\tau}{2} \Delta\left[\left(G_{n}^{\varepsilon}(\tau)+\tau f\left(\left|u_{\frac{1}{2}}\right|^{2}\right)\right) u_{\frac{1}{2}}\right] \\
& \quad-\frac{1}{2}\left(G_{n}^{\varepsilon}(\tau)+\tau f\left(\left|u_{\frac{1}{2}}\right|^{2}\right)\right)^{2} u_{\frac{1}{2}}+\mathcal{R}_{4,4}\left(V_{n}^{\varepsilon}, u, \tau^{3}\right)
\end{aligned}
$$


By subtraction, we get

$$
\begin{aligned}
u\left(t_{n+1}\right)-\Phi_{S}^{\tau, t_{n}}\left(u\left(t_{n}\right)\right) & \\
= & -\frac{\tau}{2} \Delta\left(G_{n}^{\varepsilon}(\tau) u_{\frac{1}{2}}\right)+\Delta\left(H_{n}^{\varepsilon}(\tau) u_{\frac{1}{2}}\right)+\left[\frac{\tau}{2} G_{n}^{\varepsilon}(\tau)-H_{n}^{\varepsilon}(\tau)\right] \Delta u_{\frac{1}{2}}+\mathcal{R}_{0,0}\left(u, \partial_{t t} u, \tau^{3}\right) \\
& -\left[H_{n}^{\varepsilon}(\tau)+\int_{0}^{\tau} G_{n}^{\varepsilon}(s) \mathrm{d} s-\tau G_{n}^{\varepsilon}(\tau)\right] f\left(\left|u_{\frac{1}{2}}\right|^{2}\right) u_{\frac{1}{2}}+\mathcal{R}_{4,4}\left(V_{n}^{\varepsilon}, u, \tau^{3}\right)+\mathcal{R}_{2,2}\left(u, \partial_{t} u, \tau^{3}\right) \\
= & 2 \nabla u_{\frac{1}{2}} \cdot \nabla\left[H_{n}^{\varepsilon}(\tau)-\frac{\tau}{2} G_{n}^{\varepsilon}(\tau)\right]+u_{\frac{1}{2}} \Delta\left[H_{n}^{\varepsilon}(\tau)-\frac{\tau}{2} G_{n}^{\varepsilon}(\tau)\right] \\
& +\mathcal{R}_{4,4}\left(V_{n}^{\varepsilon}, u, \tau^{3}\right)+\mathcal{R}_{2,2}\left(u, \partial_{t} u, \tau^{3}\right)+\min \left\{\mathcal{R}_{0,0}\left(u, \partial_{t} u, \tau^{2}\right), \mathcal{R}_{0,0}\left(u, \partial_{t t} u, \tau^{3}\right)\right\},
\end{aligned}
$$

where we have used the property that

$$
\begin{aligned}
H_{n}^{\varepsilon}(\tau)+\int_{0}^{\tau} G_{n}^{\varepsilon}(s) \mathrm{d} s-\tau G_{n}^{\varepsilon}(\tau) & =\int_{0}^{\tau} s V_{n}^{\varepsilon}(s) \mathrm{d} s+\int_{0}^{\tau} \int_{0}^{s} V_{n}^{\varepsilon}(y) d y \mathrm{~d} s-\tau \int_{0}^{\tau} V_{n}^{\varepsilon}(s) \mathrm{d} s \\
& =\int_{0}^{\tau}(s-\tau) V_{n}^{\varepsilon}(s) \mathrm{d} s+\int_{0}^{\tau} V_{n}^{\varepsilon}(y) \int_{y}^{\tau} \mathrm{d} s d y=0 .
\end{aligned}
$$

Noticing that

$$
H_{n}^{\varepsilon}(\tau)-\frac{\tau}{2} G_{n}^{\varepsilon}(\tau)=\int_{0}^{\tau}\left(s-\frac{\tau}{2}\right) V_{n}^{\varepsilon}(s) \mathrm{d} s=\frac{1}{2} \int_{0}^{\tau} s(\tau-s) \partial_{t} V_{n}^{\varepsilon}(s) \mathrm{d} s,
$$

which together with assumption (2.7) yields that

$$
\begin{aligned}
u\left(t_{n+1}\right)-\Phi_{S}^{\tau, t_{n}}\left(u\left(t_{n}\right)\right)= & \mathcal{R}_{4,4}\left(V_{n}^{\varepsilon}, u, \tau^{3}\right)+\mathcal{R}_{2,2}\left(u, \partial_{t} u, \tau^{3}\right)+\min \left\{\mathcal{R}_{0,0}\left(u, \partial_{t} u, \tau^{2}\right), \mathcal{R}_{0,0}\left(u, \partial_{t t} u, \tau^{3}\right)\right\} \\
& +\min \left\{\mathcal{R}_{1,2}\left(u, V_{n}^{\varepsilon}, \tau^{2}\right), \mathcal{R}_{1,2}\left(u, \partial_{t} V_{n}^{\varepsilon}, \tau^{3}\right)\right\} \\
\lesssim & \min \left\{\tau^{2}, \frac{\tau^{3}}{\varepsilon}\right\}
\end{aligned}
$$

and the proof is completed.

Combining the local error bound in Lemma 4.3 and the stability estimate (4.1), and applying a similar argument as in the proof of Theorem 2.1, we can get the error bound of the second-order scheme (2.4) as shown in Theorem 2.2.

Remark 4.4. Although our presentation stops at the Strang splitting scheme, the higher-order splitting schemes whose local truncation error certainly involves higher-order time derivatives of $V^{\varepsilon}$, are not expected to be uniformly accurate at their optimal convergence rate.

\section{Numerical RESUlt}

In this section, we present the convergence tests results of the splitting schemes for solving the NLSE-OP (1.2) and the KGE-OP (2.8) for a wide range of $\varepsilon \in(0,1]$. We implement the spatial discretization of the splitting schemes by Fourier pseudo-spectral method [24].

Example 5.1. (Test for NLSE-OP) We take one-dimensional example of NLSE-OP for test, i.e., $d=1, \mathbf{x}=x$ in (1.2). We consider the cubic nonlinearity $f(u)=-u^{3}$ and choose the initial condition for $(1.2)$ as

$$
u_{0}(x)=\frac{\sin (2 x)}{2+\cos (x)}, \quad x \in[-\pi, \pi] .
$$

We take the oscillatory potential $V^{\varepsilon}(x, t)$ as

$$
V^{\varepsilon}(x, t)=U^{\varepsilon}(x, t)+2 \cos (t) \cos ^{2}(x),
$$

where $U^{\varepsilon}(x, t)$ is the solution of the following wave equation:

$$
\left\{\begin{array}{l}
\varepsilon^{2} \partial_{t t} U^{\varepsilon}(x, t)-\partial_{x x} U^{\varepsilon}(x, t)=0, \quad x \in(-\pi, \pi), t>0, \\
U^{\varepsilon}(x, 0)=\frac{\sin (x)}{2+\cos (2 x)}+\sin (2 x), \partial_{t} U^{\varepsilon}(x, 0)=\frac{1}{\varepsilon}[\cos (3 x)+\sin (2 x)], x \in[-\pi, \pi], \\
U^{\varepsilon}(\pi, t)=U^{\varepsilon}(-\pi, t), \quad \partial_{x} U^{\varepsilon}(\pi, t)=\partial_{x} U^{\varepsilon}(-\pi, t), \quad t \geq 0 .
\end{array}\right.
$$



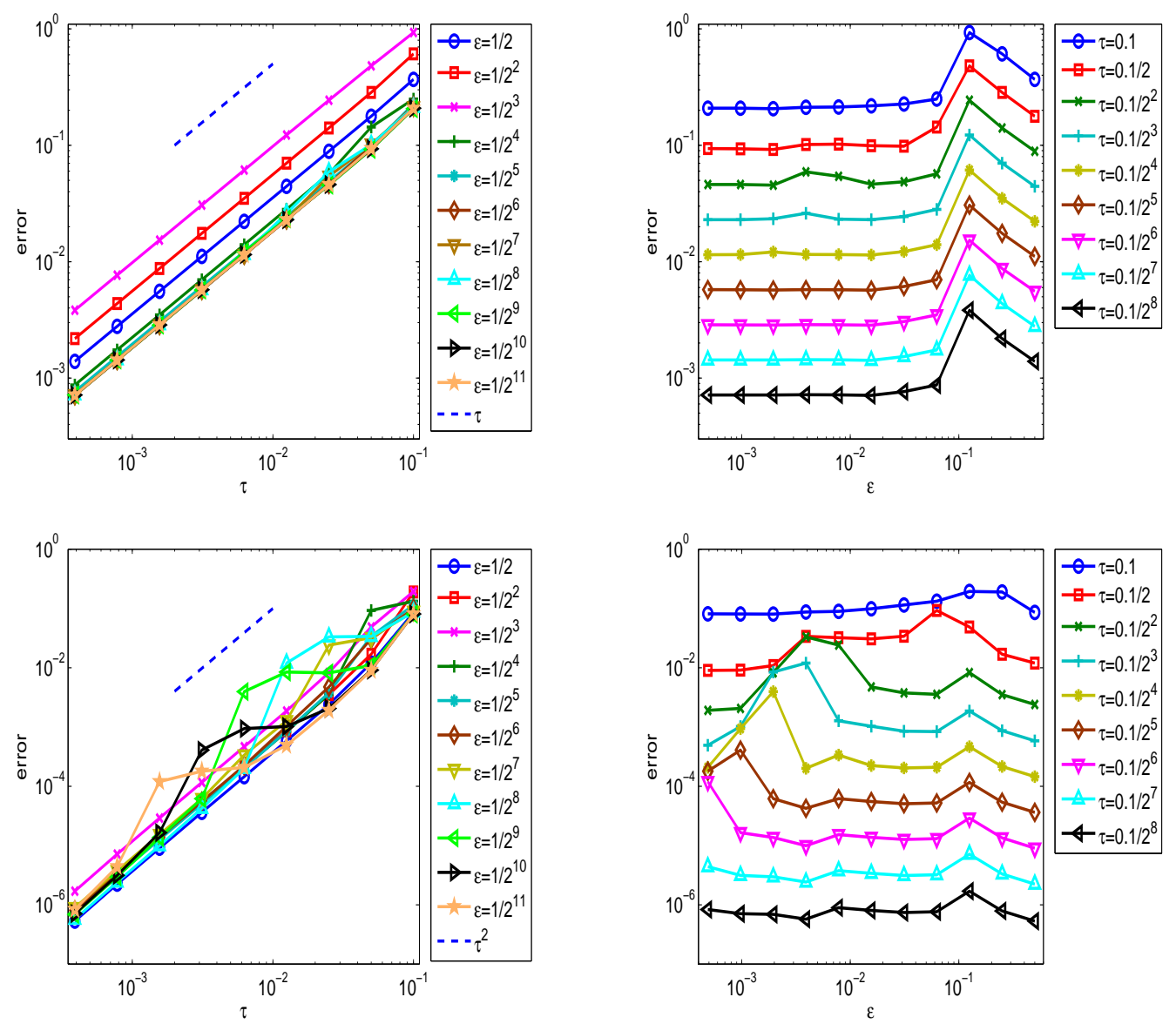

FiguRE 1. Error $\left\|u\left(\cdot, t_{n}\right)-u^{n}\right\|_{H^{1}}$ at $t=2$ of the Lie-Trotter splitting (above) and Strang splitting (below) for the NLSE-OP (1.2) under different $\varepsilon$ and $\tau$.

We solve the NLSE-OP (1.2) till $t=2$ and compute the error of splitting schemes:

$$
\left\|u\left(\cdot, t_{n}\right)-u^{n}\right\|_{H^{1}} .
$$

The reference solution is given by a 4th order Yoshida splitting scheme [28] with Fourier pseudospectral method under very small time step size $\tau=10^{-5}$ and fine mesh size $\Delta x=2 \pi / 128$. We plot the temporal error of the Lie-Trotter splitting scheme and the Strang splitting scheme under different $0<\varepsilon<1$ in Figure 1, where the spatial mesh size $\Delta x=2 \pi / 128$ is kept so that the spatial discretization error is negligible.

From the numerical results in Figure 1, we can see that

1) The Lie-Trotter splitting scheme (2.3) converges uniformly for all $\varepsilon \in(0,1]$ at the first order rate for solving the NLSE-OP (1.2).

2) The Strang splitting scheme (2.4) converges at the second order rate when the time step $\tau$ is small enough, but the accuracy is not uniform and optimal for $\varepsilon \in(0,1]$.

Example 5.2. (Test for KGE-OP) As another example, we solve the one-dimensional KGE-OP (2.8). We take a cubic nonlinearity $f(u)=u^{3}$ and initial values in (2.8) as:

$$
u_{0}(x)=\frac{\sin (2 x)}{2+\cos (x)}, \quad u_{1}(x)=\frac{1+\cos (x)}{1-\sin (2 x) / 2}, \quad x \in[-\pi, \pi] .
$$

The potential $V^{\varepsilon}(x, t)$ is chosen as same as in (5.1). 

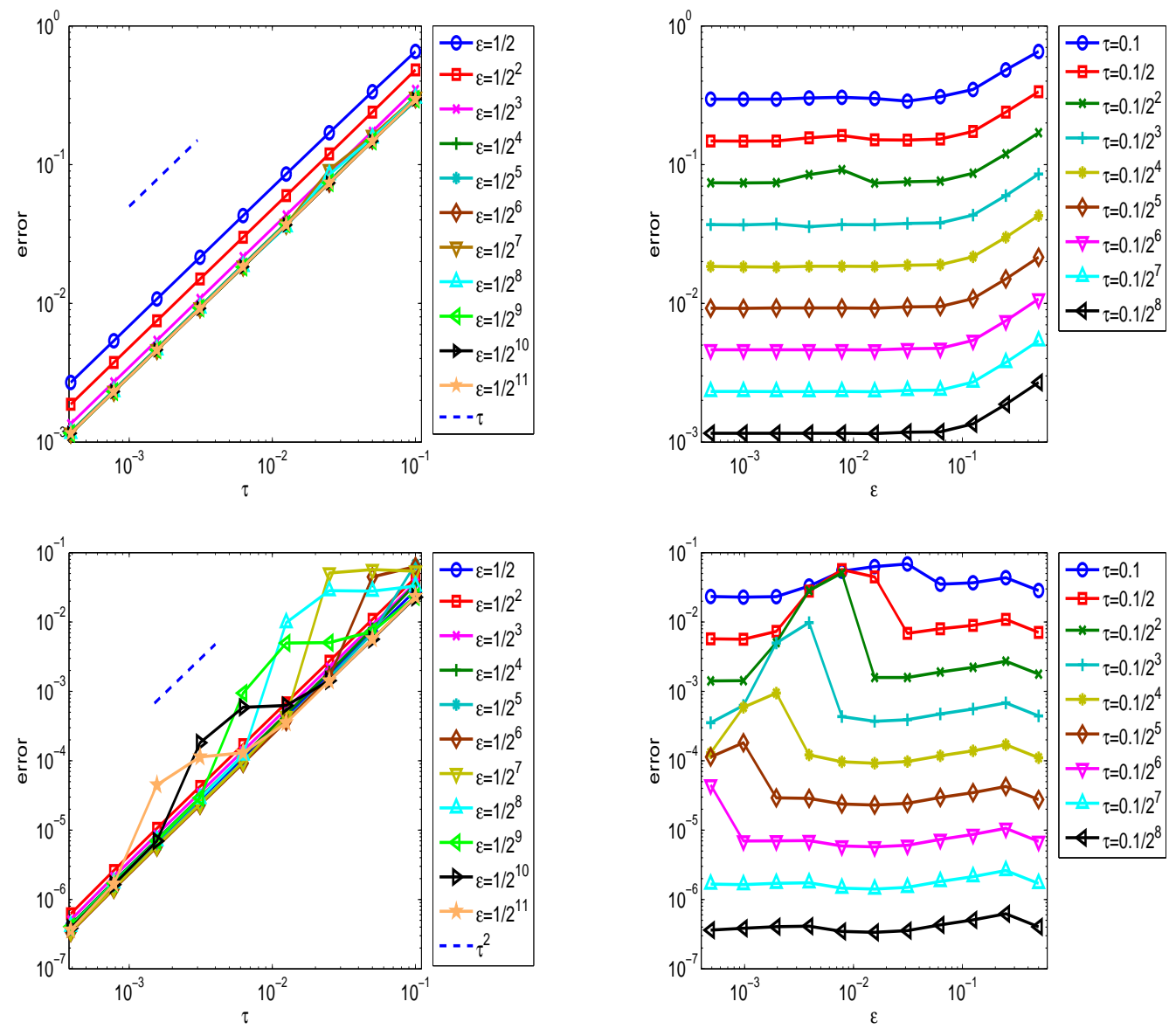

FigURE 2. Error $\left\|u\left(\cdot, t_{n}\right)-u^{n}\right\|_{H^{1}}+\left\|\partial_{t} u\left(\cdot, t_{n}\right)-\dot{u}^{n}\right\|_{H^{1}}$ at $t=2$ of the Lie-Trotter splitting (above) and Strang splitting (below) for the KGE-OP (2.8) under different $\varepsilon$ and $\tau$.

We solve the KGE-OP (2.8) till $t=2$ and compute the error:

$$
\left\|u\left(\cdot, t_{n}\right)-u^{n}\right\|_{H^{1}}+\left\|\partial_{t} u\left(\cdot, t_{n}\right)-\dot{u}^{n}\right\|_{H^{1}} .
$$

The reference solution is obtained similarly as before. Figure 2 shows the temporal error (fixed $\Delta x=2 \pi / 128)$ of the Lie-Trotter splitting scheme and the Strang splitting scheme under different $\varepsilon \in(0,1]$. As can be seen, the numerical results in Figure 2 are similar as before and hence illustrates once again the same conclusions on the convergence result of the splitting schemes. As for comparisons, we show the corresponding error from the Deuflhard-type exponential integrator [30] in Figure 3. Although the splitting methods and the exponential integrators (or known as trigonometric integrators) are closely related [10, 19], it is clear that the exponential integrator gives much worse approximations than the splitting scheme in our model case, where the error in Figure 3 increases dramatically as $\varepsilon$ becomes small. We comment that the error of the Gautschi-type exponential integrator $[3,29]$ is even worse when $\varepsilon$ is small.

\section{Conclusion}

In this work, we consider the nonlinear Schrödinger equation with a highly oscillatory potential (NLSE-OP) as our model problem, where the potential introduces fast temporal oscillations to the solution. This model problem is motivated from recent studies on multiscale problems such 

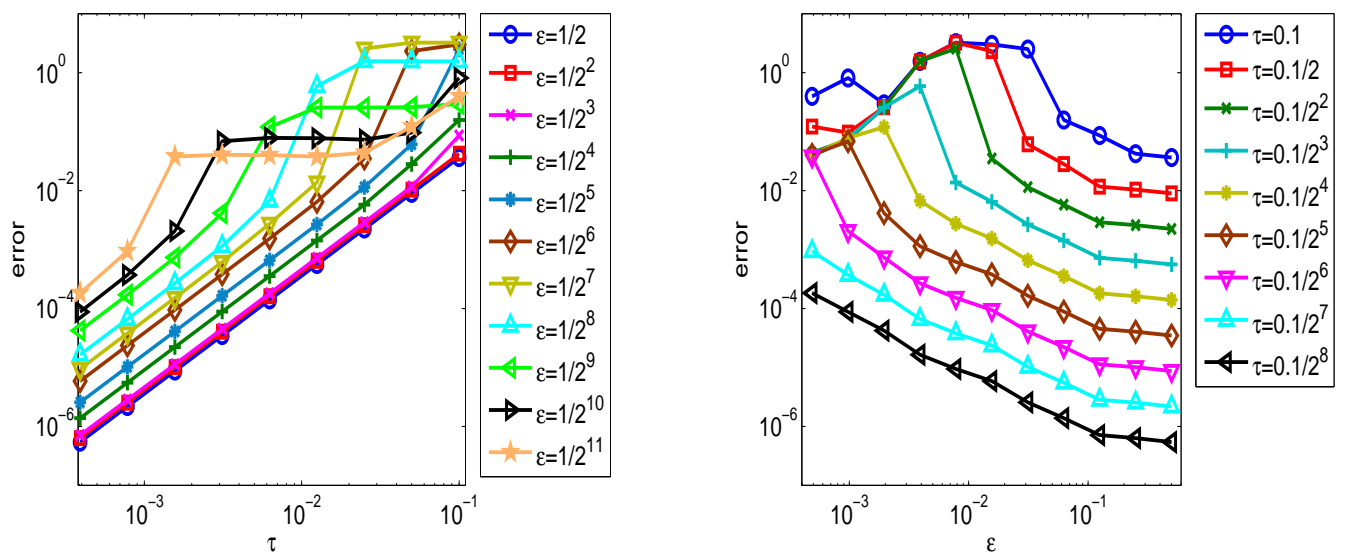

Figure 3. Error $\left\|u(\cdot, t)-u^{n}\right\|_{H^{1}}+\left\|\partial_{t}(\cdot, t)-\dot{u}^{n}\right\|_{H^{1}}$ at $t=2$ of exponential integrator for the KGE-OP (2.8) under different $\varepsilon$ and $\tau$.

as the subsonic limit of the Zakharov system. The time-splitting schemes are applied to solve the NLSE-OP, where the sub-flows are integrated exactly. We rigorously analyze the error bounds of the splitting schemes, where the results show that the Lie-Trotter splitting scheme converges linearly and uniformly with respect to the oscillation frequency from the potential, while the Strang splitting scheme is not uniformly second order accurate. Due to the exact integration of the oscillatory potential, the splitting schemes still give much more accurate approximations than the exponential integrators in the highly oscillatory regime. Extensions are made to the nonlinear Klein-Gordon equation with an oscillatory potential. Numerical results justify the theoretical estimates.

\section{ACKNOWLEDGEMENTS}

C. Su is supported by the Alexander von Humboldt Foundation. X. Zhao is supported by the French ANR project MOONRISE ANR-14-CE23-0007-01.

\section{REFERENCES}

[1] R. A. Adams, J. J. Fournier, Sobolev spaces, Elsevier, 2003.

[2] W. BAO, Y. CAI, X. ZhAO, A uniformly accurate multiscale time integrator pseudospectral method for the Klein-Gordon equation in the nonrelativistic limit regime, SIAM J. Numer. Anal. 52 (2014) pp. 2488-2511.

[3] W. BaO, X. Dong, Analysis and comparison of numerical methods for the Klein-Gordon equation in the nonrelativistic limit regime, Numer. Math. 120 (2012), pp. 189-229.

[4] W. BAO, C. Su, Uniform error bounds of a finite difference method for the Klein-Gordon-Zakharov system in the subsonic limit regime, Math. Comp. 87 (2018), pp. 2133-2158.

[5] W. BAO, C. Su, Uniform error bounds of a finite difference method for the Zakharov system in the subsonic limit regime via an asymptotic consistent formulation, Multiscale Model. Simul. 15 (2017), pp. 977-1002.

[6] W. BAO, C. Su, Uniformly and optimally accurate methods for the Zakharov system in the subsonic limit regime, SIAM J. Sci. Comput. 40 (2017), pp. A929-A953.

[7] W. Baо, X. Zhaо, A uniformly accurate multiscale time integrator spectral method for the Klein-GordonZakharov system in the high-plasma-frequency limit regime, J. Comput. Phys. 327 (2016), pp. 270-293.

[8] S. Baumstark, E. Faou, K. Schratz, Uniformly accurate exponential-type integrators for Klein-Gordon equations with asymptotic convergence to the classical NLS splitting, Math. Comp. 87 (2018), pp. 1227-1254.

[9] C. Besse, B. Bidégaray, S. Descombes, Order estimates in time of splitting methods for the nonlinear Schrödinger equation, SIAM J. Numer. Anal. 40 (2002), pp. 26-40.

[10] S. Buchholz, L. Gauckler, V. Grimm, M. Hochbruck, T. Jahnke, Closing the gap between trigonometric integrators and splitting methods for highly oscillatory differential equations, IMA J. Numer. Anal. 38 (2017), pp. 57-74.

[11] Ph. Chartier, N. Crouseilles, M. Lemou, F. Méhats, Uniformly accurate numerical schemes for highly oscillatory Klein-Gordon and nonlinear Schrödinger equations, Numer. Math. 129 (2015), pp. 211-250. 
[12] Ph. Chartier, M. Lemou, F. Méhats, G. Vilmart, A new class of uniformly accurate methods for highly oscillatory evolution equations, to appear on Found. Comput. Math. (2019).

[13] Ph. Chartier, F. Méhats, M. Thalhammer, Y. Zhang, Improved error estimates for splitting methods applied to highly-oscillatory nonlinear Schrödinger equations, Math. Comp. 85 (2016), pp. 2863-2885.

[14] N. Crouseilles, M. Lemou, F. Ménats, X. Zhao, Uniformly accurate Particle-in-Cell method for the long time two-dimensional Vlasov-Poisson equation with uniform strong magnetic field, J. Comput. Phys. 346 (2017), pp. $172-190$.

[15] N. Crouseilles, S.A. Hirstoaga, X. Zhao, Multiscale Particle-In-Cell methods and comparisons for long time two-dimensional Vlasov-Poisson equation with strong magnetic field, Comput. Phys. Commun. 222 (2018), pp. 136-151.

[16] E. FAOU, Geometric numerical integration and Schrödinger equations, European Math. Soc. 2012.

[17] L. Gauckler, Convergence of a split-step Hermite method for the Gross-Pitaevskii equation, IMA J. Numer. Anal. 31 (2011), pp. 396-415.

[18] E. Hairer, Ch. Lubich, G. Wanner, Geometric Numerical Integration: Structure-Preserving Algorithms for Ordinary Differential Equations, Springer, Berlin, 2006.

[19] Ch. Lubich, On splitting methods for Schrödinger-Poisson and cubic nonlinear Schrödinger equations, Math. Comp. 77 (2008), pp. 2141-2153.

[20] Y. MA, C. Su, A uniformly and optimally accurate multiscale time integrator method for the Klein-GordonZakharov system in the subsonic limit regime, Comput. Math. Appl. 76 (2018), pp. 602-619.

[21] N. Masmoudi, K. NAKAnishi, From the Klein-Gordon-Zakharov system to the nonlinear Schrödinger equation, J. Hyperbolic Differential Equations, 2 (2005), pp. 975-1008.

[22] N. Mauser, Y. Zhang, X. ZhaO, On the rotating nonlinear Klein-Gordon equation: non-relativistic limit and numerical methods, preprint (2018), hal-01956352.

[23] J. Shen, Z.Q. WAng, Error analysis of the Strang time-splitting Laguerre-Hermite/Hermite collocation methods for the Gross-Pitaevskii equation, Found. Comput. Math. 13 (2013), pp. 99-137.

[24] J. Shen, T. Tang, L. Wang, Spectral Methods: Algorithms, Analysis and Applications, Springer, 2011.

[25] M. Thalhammer, Convergence analysis of high-order time-splitting pseudo-spectral methods for nonlinear Schrödinger equations, SIAM J. Numer. Anal. 50 (2012), pp. 3231-3258.

[26] J.A.C. Weideman, B.M. Herbst, Split-step methods for the solution of the nonlinear Schrödinger equation, SIAM J. Numer. Anal. 23 (1986), pp. 485-507.

[27] C. Xiong, M. Good, Y. Guo, X. Liu, K. Huang, Relativistic superfluidity and vorticity from the nonlinear Klein-Gordon equation, Phys. Rev. D 90 (2014) pp.125019.

[28] H. YoshidA, Construction of higher order symplectic integrators. Phys. Lett. A 150 (1990) pp. 262-268.

[29] Y. WANG, X. ZhaO, Symmetric high order Gautschi-type exponential wave integrators pseudospectral method for the nonlinear Klein-Gordon equation in the nonrelativistic limit regime, Int. J. Numer. Anal. Model. 15 (2017), pp. 405-427.

[30] X. ZhaO, On error estimates of an exponential wave integrator sine pseudospectral method for the Klein-GordonZakharov system, Numer. Meth. Part. Differ. Equat. 32 (2016), pp. 266-291.

C. Su: Zentrum Mathematik, Technische Universität München, 85748 Garching bei München, Germany E-mail address: sucm13@163.com

X. Zhao: School of Mathematics and Statistics, Wuhan University, 430072 Wuhan, China

E-mail address: zhxfnus@gmail.com 